\title{
La Estrategia Territorial Nacional (ETN) y su ordenamiento en el Plan Toda Una Vida 2017-2021 en sus inicios de pandemia - Ecuador
}

\section{The National Territorial Strategy (ETN) and its ordering in the Toda Una Vida Plan 2017-2021 in its early stages of pandemic - Ecuador}

DOI: $10.46932 / \operatorname{sjd} \mathrm{dv} 3 \mathrm{n} 1-113$

Received in: Jan 30st, 2021

Accepted in: Feb 1th, 2022

\author{
Sheila Janet Rangel Gómez \\ Maestra en Gobierno y Gestión Local \\ Institution: Universidad Estatal de Bolívar \\ Address: Av. Ernesto Che Guevara s/n y Av. Gabriel Secaira, Guaranda, Ecuador \\ E-mail: srangel@ueb.edu.ec \\ Jorge Armando Zula Cujano \\ Doctor en Ciencias Económicas, Especialidad en Administración PhD \\ Institution: Universidad Estatal de Bolívar \\ Address: Av. Ernesto Che Guevara s/n y Av. Gabriel Secaira, Guaranda, Ecuador \\ E-mail: jzua@ueb.edu.ec \\ Fernando Fredi Rea García \\ Maestro en Gobierno y Gestión Local \\ Institution: Universidad Estatal de Bolívar \\ Address: Av. Ernesto Che Guevara s/n y Av. Gabriel Secaira, Guaranda, Ecuador \\ E-mail: frea@ueb.edu.ec \\ Vásquez Aldaz Allison Camila \\ Estudiante de la Carrera de Sociología \\ Institution: Universidad Estatal de Bolívar \\ Address: Av. Ernesto Che Guevara s/n y Av. Gabriel Secaira, Guaranda, Ecuador \\ E-mail: alvasquez@mailes.ueb.edu
}

\section{RESUMEN}

La Estrategia Territorial Nacional (ETN) en conjunto con el Plan Nacional de Desarrollo se adjudican en el ordenamiento territorial. Cada uno con diversos planteamientos, pero con una finalidad de la búsqueda del bien común. El objetivo de este artículo fue analizar como estos planes se vieron afectados por la reorganización social surgida a partir del covid-19 lo que dificulto la habitabilidad social y territorial. La investigación se realizó través del método cualitativo, en el cual se logró efectuar la recopilación bibliográfica. El ordenamiento territorial muestra la gran influencia que tiene dentro de los planes, pues es a partir de ello se da un sentido a las planificaciones que desarrollan en cuanto a políticas públicas y la organización de los espacios públicos. La Estrategia Territorial Nacional (ETN) señala cuáles son las necesidades básicas que tienen los seres humanos, relacionando a ello el cómo se organizará el territorio y esto beneficie al desarrollo individual y colectivo. El Plan de Desarrollo Nacional Ecuatoriano contribuye al cumplimiento de aspectos importantes y al progreso humano que puedan dar con la ejecución de los derechos humanos y estos persistan durante toda la vida, así mismo, mejorar la economía y tener una sociedad digna que priorice las necesidades fundamentales del país. La ETN y el PND conforman conjuntos sociales, económicos, políticos, culturales, demográficos, entre otros, lo cual se vio 
modificado por la pandemia. Cada conjunto aporta con iniciativas sustanciales para la ejecución y planificación de las políticas públicas ya que son aquellas prioridades que el gobierno busca establecer. Sin embargo, no están dentro del presupuesto y catalogados como actividad necesaria.

Palabras clave: territorio, covid-19, ordenamiento, Ecuador, ciudad.

\begin{abstract}
The National Territorial Strategy (NTS), together with the National Development Plan, are adjudicated in territorial planning. Each one with different approaches, but with the purpose of seeking the common good. The objective of this article was to analyze how these plans were affected by the social reorganization that arose from covid-19, which hindered social and territorial habitability. The research was carried out through the qualitative method, in which the bibliographic compilation was carried out. The territorial planning shows the great influence it has within the plans, since it is from it that a sense is given to the plans that are developed in terms of public policies and the organization of public spaces. The National Territorial Strategy (ETN) indicates the basic needs of human beings, relating to how the territory will be organized to benefit individual and collective development. The Ecuadorian National Development Plan contributes to the fulfillment of important aspects and human progress that can be given with the execution of human rights and these persist throughout life, as well as improving the economy and having a dignified society that prioritizes the fundamental needs of the country. The TNA and the NDP make up social, economic, political, cultural, demographic, among others, which was modified by the pandemic. Each set contributes with substantial initiatives for the execution and planning of public policies since they are those priorities that the government seeks to establish. However, they are not within the budget and catalogued as a necessary activity.
\end{abstract}

Keywords: territory, covid-19, planning, Ecuador, city.

\title{
1 INTRODUCCIÓN
}

En Latinoamérica, así como en el resto del mundo y en el caso ecuatoriano, es de suma importancia crear un Plan Nacional de Desarrollo (PND) en el que se enmarquen todas y cada una de las estrategias/proyectos y programas sociales, políticos, estatales y territoriales, con el único fin de impulsar el desarrollo del país, no sin antes proceder a su evaluación y de ahí formular una propuesta. Además, en el caso particular del Ecuador se busca cumplir con el artículo 3, número 5, en el que se detalla que se debe "planificar el desarrollo nacional, erradicar la pobreza, promover el desarrollo sustentable y la redistribución equitativa de los recursos y la riqueza, para acceder al buen vivir" Secretaria Nacional de Planificación y Desarrollo (Senplades, 2017, p. 16) Una de las disciplinas modernas para la organización y planificación del territorio es el ordenamiento territorial que se aplica en los territorios rurales y urbanos, siendo este ultimo el caso que se abordará.

\section{MATERIALES Y MÉTODOS}

El método empleado es el método cualitativo utilizando como herramienta la recolección de bibliografía que acorde a Vera (s.f.) definen a la metodología cualitativa de la siguiente manera: 
es aquella donde se estudia la calidad de las actividades, relaciones, asuntos, medios, materiales o instrumentos en una determinada situación o problema. La misma procura por lograr una descripción holística, esto es, que intenta analizar exhaustivamente, con sumo detalle, un asunto o actividad en particular. (p. 1)

Esta herramienta permitió identificar las principales corrientes críticas - teóricas - conceptuales del ordenamiento territorial y la ciudad como eje central de desarrollo. Además, permite observar las cualidades y necesidades llámense sociales, demográficas, ecológicas entre otros que son propias del territorio ecuatoriano para realizar un análisis y en consecuencia de ello la creación del Plan Nacional de Desarrollo, para finalizar con una breve discusión sobre su reordenamiento en pandemia.

\section{RESULTADOS}

\subsection{EL ORDENAMIENTO TERRITORIAL Y SU DEFINICIÓN}

El ordenamiento territorial es una disciplina y una línea teórica del territorio que constituye una forma científica y técnica de abordarlo, pero también funge como una manera de impulsar políticas públicas y una técnica administrativa para la organización del espacio público de las ciudades y localidades, sean estas de índole urbana o rural. Se puede definir como "[...] un proceso que permite organizar las actividades y recursos en el territorio de acuerdo con las estrategias de desarrollo socioeconómico, en armonía con las particularidades geográficas y culturales. El ordenamiento territorial es obligatorio para todos los niveles de gobierno”. (Secretaría Técnica Planifica Ecuador, 2019, p. 3)

Es así como en el Ecuador, a través de una determinada planificación se organiza el territorio para el cumplimiento de diferentes funcionalidades, todas y cada una a favor de los ciudadanos con el único fin de precautelar el desarrollo socioeconómico y cultural de la ciudadanía ecuatoriana, recalcando que esta planificación se reestructura cada periodo presidencial.

\subsection{LA ESTRATEGIA TERRITORIAL NACIONAL (ETN) Y EL PLAN NACIONAL DE DESARROLLO (PND) 2017-2021-TODA UNA VIDA}

En el Ecuador y de acuerdo con la Constitución de la República en su artículo 280 se afirma que el instrumento para llevar a cabo toda la planificación y desarrollo del país es:

El Plan Nacional de Desarrollo es el instrumento al que se sujetarán las políticas, programas y proyectos públicos; la programación y ejecución del presupuesto del Estado; y la inversión y la asignación de los recursos públicos; y coordinar las competencias exclusivas entre el Estado central y los gobiernos autónomos descentralizados. Su observancia será de carácter obligatorio para el sector público e indicativo para los demás sectores. (Senplades, 2017, p. 5)

Es así, que uno de los ejes del Plan Nacional de Desarrollo es el referente a la Estrategia Territorial Nacional (ETN), en el que se identifican las necesidades de los ciudadanos ecuatorianos sea cual sea el 
lugar en el que se encuentren y posteriormente decidir qué hacer, cómo hacerlo y quiénes serán los responsables de cada acción a ejecutar en territorio, con el fin de mejorar las condiciones de vida de las familias. El ETN también,

mantiene una estructura dinámica y proactiva hacia la gestión del espacio físico y de las actividades humanas sobre este; la ETN (2017) implica la construcción de lineamientos que favorezcan la articulación, coordinación y prelación de instrumentos de planificación, gestión y ordenamiento territorial. Estas tendencias responden a una necesidad de dar seguimiento permanente a las políticas, actividades, requerimientos, intereses e impactos en referencia a los aspectos económicos, sociales y ambientales del territorio. Es por ello por lo que la planificación del desarrollo y del ordenamiento territorial constituye parte de un proceso continuo y cíclico, que puede tener diversas fases de formulación y alineamiento a partir de procesos permanentes de monitoreo, seguimiento y evaluación. El objetivo es orientar las intervenciones hacia una mejor calidad de vida de la población, mediante la definición de políticas, programas y proyectos de aplicación en el territorio, que contribuyan a una asignación equitativa y transparente de los recursos públicos. (Senplades, 2017, p. 110)

El territorio nacional ecuatoriano esta comprendido por tres espacios geográficos, los cuales son: la cordillera de los andes, el archipiélago de las Galápagos y la plataforma marina. A su vez, por ecorregiones ${ }^{1}$ que pueden simplificarse en región sierra, costa, amazónica y Galápagos o región insular.

El modelo territorial actual en términos sociales analiza el Índice de pobreza multidimensional (IPM) que de acuerdo con la Organización de las Naciones Unidas (ONU) consiste en un conjunto de elementos evaluados que contemplan un estado de vulneración de derechos de los individuos y agrupa cuatro dimensiones: i) educación, ii) trabajo y seguridad social, iii) salud, agua y alimentación, y iv) hábitat, vivienda y ambiente sano. En este sentido, en cuanto al índice ya indicado, las provincias de la Amazonia, Esmeraldas, Bolívar, Cotopaxi, Chimborazo, Manabí, Los Ríos, Cañar, Azuay y Loja presentan un alto índice de pobreza multidimensional, muy en especial el primer grupo mencionado. Lo que refiere a las provincias de Guayas, Carchi y Santa Elena presentan niveles inferiores al índice nacional, sin embargo, se exceptúan regiones cercanas a la frontera con Colombia.

Aunado a esto, “en el país, la población predominante es mestiza, hispanohablante (82,2\% del total de habitantes). En el mismo territorio cohabitan 14 nacionalidades y 18 pueblos, algunos de ellos con lenguas propias". (Senplades, 2017, p. 114) con esto, se afirma que la planificación de los territorios se basa en los principios de plurinacionalidad e interculturalidad, que concuerdan con la incidencia mayoritaria de la pobreza sobre los grupos humanos antes mencionados.

En términos productivos, a nivel nacional se identifican cuatro espacios diferenciados en función de los índices de producción primaria, secundaria y terciaria:

\footnotetext{
${ }^{1}$ Según el Sistema Nacional de Clasificación de Ecosistemas del Ecuador "se distinguen 87 ecosistemas, estos se identifican mediante la utilización de criterios fisionómicos, ecológicos y florísticos, en combinación con variables biogeográficas y bioclimáticas". (Ministerio del Ambiente, 2013, p. 10)
} 
- La primera categoría: "Desarrollo industrial en crecimiento con servicios terciarios especializados" se encuentra marcada por la bicefalia histórica entre las mayores ciudades del país -Quito y Guayaquil-, las cuales marcan el desarrollo en industria, manufactura y servicios especializados.

- La segunda categoría se denomina "Desarrollo primario consolidado con enclaves industriales básicos y/o turísticos" y se caracteriza por tener un nivel de diversificación primaria productiva elevada y consolidada, lo cual permite incursionar en niveles básicos de industrialización, manufactura y/o turismo.

- Una tercera categoría establecida como "Bajo desarrollo primario de mayor diversificación con puntales productivos" se encuentra en la mayor superficie del territorio nacional. Tiene una gran presencia en la zona norte del país - exceptuando la ciudad de Quito y su ámbito de influencia- y en el entorno petrolero circunscrito principalmente a las ciudades de Nueva Loja, Francisco de Orellana y Shushufindi.

- La cuarta categoría de "Bajo desarrollo primario poco diversificado" se encuentra en las estribaciones externas de la cordillera oriental, con un débil impulso económico en los tres sectores. (Senplades, 2017, pp. 114 - 115)

En cuanto a las problemáticas ambientales las provincias con mayor contaminación, sobreutilización de suelos, recurso hídrico y deforestación son: Esmeraldas, Bolívar, Cotopaxi, Chimborazo, Manabí, los Ríos, el Oro, Guayas, Manabí y Santo Domingo, mientras que Galápagos, amazonia central y una zona del Guayas presentan un potencial de desarrollo debido a la presencia de una diversidad de flora y fauna, además de el espacio marino que ofrece ámbitos de exploración y descubrimiento que permitan desarrollar las potencialidades del desarrollo económico, social y laboral. No obstante, el Ecuador, cuenta con amenazas volcánicas que ponen en riesgo el territorio y sus habitantes.

Como parte del sistema territorial ecuatoriano, se presenta la red de asentamientos humanos conocidas como metrópolis y articuladores nacionales y regionales, con su entorno y ubicación, los cuales responden a una lógica social, económica, ambiental, cultural e histórica. El primero, se aglomera dentro del desarrollo industrial, en el que se han agrupado la gran mayoría de industrias que agregan valor, así como los dedicados a servicios terciarios especializados; en segundo lugar, se asocian a grupos dedicados a actividades alrededor de la extracción de recursos naturales no renovables, pesca o turismo. En tercer lugar, en el Ecuador se apuesta por la desconcentración y descentralización en el territorio y este se enfocó hacia la prestación de servicios públicos cercanos a la ciudadanía.

Con todo lo anterior, Senplades (2017) generó una propuesta para crear directrices y lineamientos territoriales que se detallan en la tabla 1 que se encuentra a continuación. 
Tabla 1 Propuesta de lineamientos territoriales del Plan Nacional de Desarrollo 2017-2021.

\section{LINEAMIENTOS}

a) Reducción de inequidades sociales y territoriales: Promover programas de vivienda de interés social en suelo urbano vacante y zonas provistas de servicios públicos y equipamiento básico, evitando la segregación espacial y la expulsión de la población residente y evaluando adecuadamente el riesgo de ocurrencia de desastres de origen natural o antrópico.

b) Gestión del hábitat para la sustentabilidad ambiental y la gestión integral de riesgos: Planificar el desarrollo económico sobre la base de la compatibilidad de usos y la vocación territorial, sus diversidades culturales y sus dinámicas socio-ambientales.

c) Fortalecimiento de un sistema de asentamientos humanos policéntricos, articulados y complementarios: Afianzar procesos de planificación, regulación y gestión urbano-ambiental, priorizando la construcción social del hábitat y la función social de la ciudad y la propiedad.

d) Impulso a la productividad y la competitividad sistémica a partir del potenciamiento de los roles y funcionalidades del territorio: Planificar el desarrollo económico sobre la base de la compatibilidad de usos y la vocación territorial, sus diversidades culturales y sus dinámicas socioambientales.

e) Consolidación de modelos de gestión descentralizada y desconcentrada, con pertinencia territorial: Articular y complementar los procesos de desconcentración y descentralización para la gestión multinivel de los bienes y servicios públicos.

f) Articulación de instrumentos del Sistema Nacional Descentralizado de Planificación Participativa: Fortalecer las capacidades institucionales que favorezcan la articulación, coordinación y prelación de los instrumentos de planificación y ordenamiento territorial con enfoque de riesgos, así como la incorporación de áreas geográficas con requerimientos específicos y de manejo especial.

g) Fomento a mecanismos de asociatividad multiactor y multinivel para la gestión y la gobernanza territorial: Incentivar la organización social, libre, autónoma, inclusiva y responsable en organizaciones de base territorial.

Nota: Elaboración propia, con base en el Plan Nacional de Desarrollo 2017-2021- Toda una vida.

Lineamientos de articulación para la gestión territorial y gobernanza multinivel 
Figura 1. Metas de las Estrategias Territoriales Nacionales, 2017-2021.

Meta 1. Elevar los niveles de

suficiencia vial de la red

nacional al menos a valores

mayores al promedio nacional

del Índice de Engel.
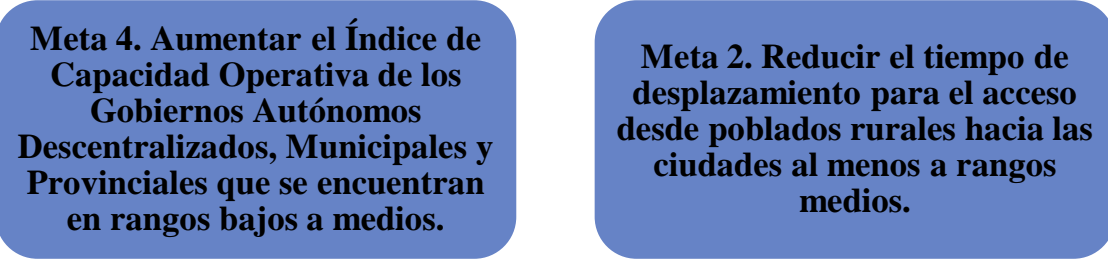

Meta 3. Incrementar el Índice

de Prosperidad de Ciudades en

al menos 2 de sus dimensiones,

en aquellas ciudades que se

encuentran con factores de

prosperidad débiles.

Nota: Elaboración propia, con base en el Plan Nacional de Desarrollo 2017-2021- Toda una vida.

Las metas y objetivos con anterioridad fueron ejecutados a partir de una planificación derivada, en concordancia con la política pública actual y fueron regulados por la Senplades (2017), además,

se enfatiza en la competencia concurrente de la planificación y el ordenamiento territorial en los diferentes niveles de gobierno y se dispone la formulación/armonización e implementación de los siguientes instrumentos que forman parte del Sistema Nacional Descentralizado de Planificación Participativa, que son regulados por la legislación vigente. (p. 127)

De esta manera, la coordinación territorial con los diferentes niveles de gobierno fue a través de Agendas de Coordinación Intersectorial, Planes sectoriales, planes Institucionales, Agendas Nacionales para la Igualdad, Agendas de Coordinación Zonal, Planes de desarrollo y ordenamiento territorial de los Gobiernos Autónomos Descentralizados, Planes especiales para proyectos nacionales de carácter estratégico, Planes territoriales diferenciados, Planes fronterizos y Programas y proyectos, todos ellos coordinados mediante un Sistema de Información Territorial y un constante seguimiento y evaluación del Plan Nacional de Desarrollo y de la Estrategia Territorial.

\section{DISCUSIÓN}

\subsection{EL EJE DE PLANEACIÓN DEL ORDENAMIENTO TERRITORIAL: LAS CIUDADES}

La ciudad es un espacio geográfico determinado en donde habitan un sin número de personas, en las que su cantidad es bastante dispareja y no siempre es un sitio adecuado para muchas de ellas. Sin embargo, no siempre está dentro de las características normales para considerarlo como tal, ya que existen 
una multiplicidad de territorios y con características propias. Así mismo, en ella coexisten varias culturas que realizan una diversidad de funciones, actividades diarias propias de su cotidianidad y que se encuentran enmarcadas dentro de los ejes económicos-productivos, sociales y políticos entre otras particularidades en torno al espacio.

Bottino (2009) hace referencia a la ciudad como:

[...] un espacio complejo, su dinamismo, sus habitantes tan dispares, sedentarios, sus funciones, las actividades tan diversas que en ellas se llevan a cabo, hacen que, definir qué es un espacio urbano no sea tarea sencilla; se pueden tomar criterios variados, como ser: numérico, legales, funcionales, administrativos, pero siempre vamos a encontrarnos con muchas definiciones según el criterio adoptado. (p. 1)

Por lo tanto, las ciudades son grandes extensiones geográficas en donde están inmersas aglomeraciones de habitantes y se consideran de este modo ya que son marcados históricamente desde décadas pasadas. La ciudad es principalmente un espacio urbano, pero indudablemente se sale de su espacio habitual ya que está presta cada día a nuevos cambios y nuevas necesidades conforme se requieran. Así mismo, es aquel espacio habitado en el que los seres humanos pueden desenvolverse con toda libertad, ya que su estructura y configuración física se adapta para el cumplimiento de las necesidades básicas y fisiológicas que una persona debe tener.

Es así como el territorio, durante las últimas décadas va más allá de un espacio físico, es un espacio de interacción en donde la sociedad se encuentra inmersa en lo económico, político, ambiental y social. Es por ello por lo que, el ordenamiento territorial es clave en la sociedad, pues cada espacio territorial ha sido designado en función de varias características como se mencionaba con anterioridad. El ordenamiento territorial entonces es "un proceso político-técnico-administrativo orientado a la organización, planificación y gestión del uso y ocupación del territorio, en función de las características biofísicas, culturales, socioeconómicas y político-institucionales” (Basso et al., citado por Méndez y Pascale, 2014, p. 6)

Si bien es cierto, el ordenamiento territorial se encarga de dividir, planificar y ubicar territorios, ya sean urbanos o rurales. Para ello, se debe conocer con anterioridad el espacio y condiciones en las que se encuentra para ser reconocido como territorio definido, el mismo debe beneficiar a individuos y colectividad como tal. Uno de los principales objetivos es mejorar la calidad de vida, de manera que la economía de estos mejore y se generen nuevas normas de socialización territorial. Adaptándose a ello, la habitabilidad deber estar dentro de los recursos sociales que un territorio pueda comprender, siendo parte de una organización estructural y social del mismo.

El ordenamiento territorial según Méndez y Pascale (2014) es necesario porque: 
[...] un municipio tiene por objeto lograr una distribución territorial más equitativa y eficiente de las oportunidades de desarrollo orientando las relaciones entre el territorio y sus ocupantes, vinculando las actividades humanas, productivas y el espacio, con el fin último, de mejorar la calidad de vida de las generaciones presentes y futuras. Las transformaciones en el sistema social, económico, cultural y político generan una serie de cambios en el uso y organización del territorio, que se deben resolver mediante el diálogo y la búsqueda de soluciones planificadas que tengan en cuenta los intereses de los sectores involucrados. (p.6)

En la actualidad, la pandemia mundial golpeó fuertemente al mundo, afectó con una gran magnitud a aspectos principalmente políticos, económicos, culturales y sociales. Por lo que, las sociedades se vieron afectadas de modo que, se modificaron actividades en cuanto a su vida diaria o cotidiana. Pues, se emplearon nuevos modos de vivencia en todas las partes del mundo. Así mismo, trajo consigo secuelas para las personas, afectando principalmente al sistema nervioso y provocando trastornos mentales psicológicos y, por ende, un reordenamiento territorial y un nuevo gobierno que se planteo un nuevo PND.

Las restricciones de los movimientos fueron el principal método de distanciamiento entre personas. El Programa de Naciones Unidas para los Asentamientos Humanos (UN-HABITAT, s. f.) hace referencia que "claramente, los espacios públicos deben ser parte de la respuesta al virus, ya sea para limitar la propagación del virus o para proporcionar formas para que las personas se relajen o lleven a cabo su sustento.” (p. 1). Sin embargo, varios espacios públicos fueron modificados y otros creados, ya que el distanciamiento debía ser de aproximadamente dos metros entre individuos. De tal modo que el ordenamiento territorial en tal caso fue reestructurado en la mayoría de las ciudades del país con fin de acoplarse a cada uno de los lineamientos establecidos por el gobierno a través de planes de ordenamiento territorial emergentes.

El distanciamiento físico fue una de las primeras medidas adoptadas para las personas, lo cual requiere que cada una de ellas tengan su propio espacio y sea adecuado en los espacios públicos. Indudablemente esto suele suceder ante todo en los países en desarrollo, mientras que en los barrios pequeños o marginales no se acatan a ciertas medidas por que el espacio es reducido. Es por ello que, ciertos sectores no suelen ser parte de las planificaciones territoriales por la distancia que tienen con relación a la ciudad.

UN-HABITAT (s. f.) da a conocer sobre cómo deben ser los espacios públicos y para ello mencionan que:

Los espacios públicos deben ser multifuncionales y adaptables. Por ejemplo, se pueden establecer mercados temporales de alimentos en espacios tales como áreas de estacionamiento para descongestionar los mercados existentes. Los pequeños espacios del vecindario se pueden transformar en áreas emergentes de centros de salud comunitarios para la distribución de alimentos o huertos. El uso compartido de calles y espacios puede permitir la venta ambulante organizada en determinados días u horas del día o para actividades de ocio como mostrar películas u obras de teatro o realizar clases de ejercicio. (p. 1) 
Entonces, el nuevo ordenamiento de los espacios públicos cambió a raíz de la pandemia mundial. Tanto espacios abiertos como lo son parques, plazas, calles, o espacios cerrados como bibliotecas públicas o centros comunitarios que han tomado nuevas medidas de seguridad para el público. A su vez, las condiciones ecológicas propias del Ecuador que se acentúan en el invierno y que acarrea desastres naturales no previstos. Todo ello hace que se erijan nuevos modos de utilización del territorio, teniendo en cuenta que no es permitido la aglomeración. Por otro lado, las nuevas normas de convivencia para los espacios afectan en gran mayoría a pequeños emprendedores que generan ingresos día a día en aquellos sectores.

Sin embargo, los espacios públicos crecen, ya que, al no permitir aglomeraciones en sitios públicos el gobierno en turno debe de accionar planes emergentes para evitar los efectos negativos sociales y económicos. Tras la pandemia mundial los gobiernos y estados optan por nuevos ordenamientos territoriales de espacios públicos, teniendo en cuenta los cambios ocasionados por la nueva distribución territorial y el aumento poblacional que a su vez genera un incremento de asentamientos humanos sin medir las consecuencias sociales y sin un estudio previo de la geografía del territorio. Si bien el Ecuador no fue el único país en no están preparado para un cambio radical por la pandemia, pero es importante incluir en los siguientes PND un análisis transversal no solo del aspecto socioeconómico de los territorios sino del aspecto cultural, de salud, educación, entre otros que ayuden a la reactivación económica de los sectores mas vulnerados.

\section{CONCLUSIÓN}

En conclusión, las ETN conforman un conjunto de elementos, sociales, culturales, históricos, medioambientales, demográficos e institucionales que necesitan encontrar una sincronización que les permita el logro de las metas planteadas, sobre todo en el cumplimiento de las agendas en los diferentes ordenes gubernamentales, así como el constante seguimiento de la evaluación al PND y al ETN y un vaciado persistente de datos al Sistema de Información Territorial, esto, permitirá la ejecución correcta de la planificación presupuestaria para la asignación de recursos públicos y la inversión pública en concordancia con las políticas públicas; dichos recursos deben de ser consistentes con la conducción sostenible, eficiente y transparente de las finanzas públicas, a partir de los requerimientos determinados en la planificación nacional y con base en las necesidades sociales de la población ecuatoriana.

Finalmente, la coordinación de todos los sectores beneficiara en la ejecución de las inversiones públicas y privadas, que fomenten el cumplimiento de las metas del PND, pero que lamentablemente requieren de fuentes de financiamiento adicionales al Presupuesto General del Estado. Se prevé incitar el desarrollo y ejecución de un plan y unas estrategias como método de mejoramiento de la calidad de vida 
de muchas personas y familias que han sido perjudicadas en la actualidad debido a grandes problemáticas principalmente de la salud. Los gobiernos siguientes y el actual deben de embarcarse en la búsqueda constante del desarrollo de los ecuatorianos a través de estrategias territoriales con mediciones geográficas, culturales, poblacionales y ecológicas que permitan realizar cruces y tomar mejores decisiones que conlleven a un verdadero desarrollo integral de todas las aristas necesarias para la población. 


\section{REFERENCIAS}

Bottino, R. (2009). La ciudad y la Urbanización. Estudios Históricos, (2), pp. 1-14.

Méndez, H., y Pascale, C. (2014). Ordenamiento Territorial en el Municipio: una guía metodológica. Recuperado el 03 de febrero del 2021 de: https://inta.gob.ar/sites/default/files/script-tmp-inta__guia_metodologica-ordenamiento_territorial_en_.pdf

Programa de Naciones Unidas para los Asentamientos Humanos, UN-HABITAT. (s.f). Espacio público $y$ COVID-19. Recuperado el 03 de febrero del 2021 de: https://unhabitat.org/sites/default/files/2020/06/spanishfinal_public_space_key_messages_covid19.pdf

Secretaria Nacional de Planificación y Desarrollo, Senplades. (2017). Plan Nacional de Desarrollo 20172021. Toda una Vida. Recuperado el 02 de febrero de 2021 de: https://www.planificacion.gob.ec/wpcontent/uploads/downloads/2017/10/PNBV-26-OCT-FINAL_0K.compressed1.pdf

Secretaría Técnica Planifica Ecuador. (2019). Plan de Desarrollo y Ordenamiento Territorial (PDOT). Recuperado el 02 de febrero del 2021 de: https://www.planificacion.gob.ec/wpcontent/uploads/downloads/2019/08/Folletos-autoridades-provinciales.pdf

Vera, L. (s.f). La investigación cualitativa. Recuperado el 02 de febrero del 2021 de: http://www.trabajosocial.unlp.edu.ar/uploads/docs/velez_vera_investigacion_cualitativa_pdf.pdf 